\title{
Adaptation of a Shared Decision-Making Tool for Early Rheumatoid Arthritis Treatment Decisions with Indigenous Patients
}

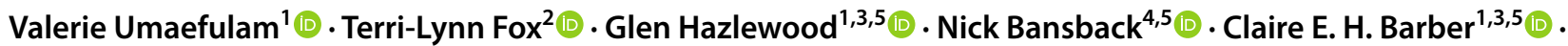 \\ Cheryl Barnabe ${ }^{1,3,5}$ (1)
}

Accepted: 22 August 2021 / Published online: 6 September 2021

(c) The Author(s) 2021

\begin{abstract}
Background Patient decision aids (PtDAs) enable shared decision-making between patients and healthcare providers. Adaptations to PtDAs for use with populations facing inequities in healthcare can improve the relevancy of information presented, incorporate appropriate cultural context, and address health literacy concerns. Our objective was to adapt the Early RA (rheumatoid arthritis) PtDA for use with Canadian Indigenous patients.

Methods The Early RA PtDA was modified through an iterative process using data obtained from semi-structured interviews of two sequential cohorts of Indigenous patients with RA. Interview data were analyzed using thematic analysis.

Results Seven participants provided initial feedback on the existing PtDA. The modifications they suggested were made and shared with another nine participants to confirm acceptability and provide further feedback. The first cohort suggested revisions to clarify medical and cost coverage information, include Indigenous traditional healing practice options, simplify text, and include Indigenous images and colors aligned with Canadian Indigenous community representation. Additional revisions were suggested by the second cohort to increase the legibility of the text, insert more Indigenous imagery, address formulary coverage for non-status First Nations patients, and include information about lifestyle factors in managing RA. Conclusion Incorporating Indigenous-specific adaptations in the design of PtDAs may increase use and relevancy to support engagement in treatment decisions, thereby supporting health-equity oriented health service interventions. Indigenous patient-specific evidence and translation of key words into the end-users' Indigenous languages should be included for implementation of the PtDA.
\end{abstract}

Cheryl Barnabe

ccbarnab@ucalgary.ca

1 Department of Medicine, Cumming School of Medicine, University of Calgary, Calgary, Alberta, Canada

2 Calgary, Alberta, Canada

3 Department of Community Health Sciences, Cumming School of Medicine, University of Calgary, Calgary, Alberta, Canada

4 Centre for Health Evaluation and Outcome Sciences at St. Paul's Hospital and University of British Columbia, Vancouver, British Columbia, Canada

5 Arthritis Research Canada, Richmond, British Columbia, Canada

\section{Key Points for Decision Makers}

Culturally appropriate and relevant decision-making tools for Indigenous patients to support patient-focused care are proposed as a way to reduce inequities in rheumatoid arthritis care.

The study advances knowledge of the features to be addressed and included in patient decision aids to enhance engagement, acceptability, and relevance for Indigenous patients. 


\section{Introduction}

Various approaches have been utilized to facilitate meaningful conversations between patients and healthcare providers that promote informed shared decision-making (SDM). One approach is to use patient decision aids (PtDAs), which are tools that explain why a decision needs to be made, present options for treatment and the benefits and harms of each, clarify values, and elicit preferences for moving forward [1]. Decision aids have been demonstrated to improve knowledge of conditions, increase patient participation in care, and reduce decisional conflict [2].

Several PtDAs have been developed to promote SDM in patients with rheumatoid arthritis (RA) for different contexts in the treatment pathway, with encouraging results [3, 4]. We have previously developed an Early RA PtDA [5] with patient partners, based on prototypes $[6,7]$ and data from a network meta-analysis [8] and following standards of the International Patient Decision Aid Standards (IPDAS) Collaboration criteria [9]. The decision aid was intended for use by individuals 16 years and older with newly diagnosed active RA choosing an initial disease-modifying treatment. Despite the many benefits of PtDAs assisting decisionmaking and furthering patient-centered care, there is limited literature on PtDAs developed for and with members of vulnerable populations [10]. While the Early RA PtDA was reviewed by patients and healthcare providers across Canada, explicit data on the ethnicity of those providing input were not collected, and thus the tool may have limited applicability to specific populations in the country, in particular, persons of the Indigenous populations in Canada who face extreme disparities in RA prevalence and outcomes [11, 12].

Generic SDM tools may not meet population-specific requirements for acceptability, health literacy, or cultural relevance [13]. This is supported by two previous studies conducted with Indigenous peoples in Canada. An existing PtDA (the Ottawa Decision Support Framework) required cultural adaptations for use by urban Indigenous women [14], and an SDM strategy called "Not Deciding Alone" grounded in Inuit epistemology has been developed in collaboration with Inuit people for use in cancer care [15]. However, to our knowledge, there is no available PtDA developed with and for Indigenous patients living with RA. Leveraging the existing Early RA PtDA (which is populated with the most recent research evidence in preparation for the Canadian Rheumatology Association's 2021 RA Guidelines), previous research to understand factors in decision making for RA treatment of Indigenous peoples [16], and a needs assessment confirming the importance of SDM in the Indigenous population with RA [17], the objective of this study was to adapt the existing Early RA PtDA for use by Indigenous patients at the point of care.

\section{Methods}

\subsection{Research Team}

Three of the authors are female Indigenous health researchers. VU is an experienced qualitative researcher, TLF identifies as a First Nations person with lived experience of $\mathrm{RA}$, and $\mathrm{CB}$ is a Métis rheumatologist and health services researcher. GH and CEHB are rheumatologists and, along with NB, are health service researchers developing, testing, and working to implement PtDAs in RA care.

\subsection{Patient Cohorts}

Inclusion criteria included Indigenous identity with a physician diagnosis of RA and being 18 years old or older. Based on the guideline for involving participants for SDM tool development [18], participants were recruited until saturation was reached. Two cohorts of patients were recruited for this iterative adaptation.

\subsubsection{Cohort 1}

The first cohort included patients recruited from three urban rheumatology clinics that provide services to Indigenous patients in Alberta, Canada. Two of these clinics were general rheumatology clinics, and the third was a specialty rheumatology clinic located in an urban Indigenous primary healthcare center.

\subsubsection{Cohort 2}

The second cohort was recruited uniquely through the specialty rheumatology clinic located at the urban Indigenous primary healthcare center.

\subsection{Process and Data Collection}

The Early RA PtDA was modified through an iterative process of feedback received through semi-structured interviews with the lead author (VU). Throughout, the IPDAS framework checklist [9] guided the tool adaptation.

\subsubsection{Cohort 1 Process}

The initial data collected were from a needs assessment study conducted with Indigenous patients living with RA by VU, TLF, and CB [17]; the results we present here are specific to feedback on the decision aid received during those interviews and are not reported in the publication. The patients were recruited from January 2020 to March 2020, with data collected by in-person interview at the medical 
clinic $(n=7)$. Participants were presented with the previously developed Early RA PtDA [5]. They were requested to provide feedback on the tool's layout, content, information gaps, and potential applications. The research team made initial adaptations based on their input. All interviews lasted between 15 and 45 min and were recorded and transcribed verbatim.

\subsubsection{Cohort 2 Process}

We then recruited a second cohort of participants to affirm that the changes made were appropriate and the content was clear, and we requested they comment on any other aspects that should be modified. The second cohort was recruited between December 2020 and March 2021. An interview guide (Online Resource 1, see the electronic supplementary material) informed by the cultural adaptation of another SDM tool with an Indigenous population [14] was developed to assist in guiding data collection. The semi-structured interviews were conducted over video conference $(n=5)$ or telephone $(n=4)$. All interviews lasted between 15 and 45 min and were recorded and transcribed verbatim.

\subsection{Data Analysis}

The data were managed using NVivo 12 (QSR International). Inductive thematic analysis [19] was conducted with an Indigenous patient partner (TLF). VU and TLF coded the transcripts, analyzed and categorized specific statements in the data, and provided the initial themes. VU reviewed the themes to ensure that they accurately were representative of the data and, following that, summarized the themes and suggested modifications of the Early RA PtDA, which was refined by CB and TLF.

\section{Results}

\subsection{Participant Demographics}

Cohort 1 included seven Indigenous women living with RA aged 37-61 years, and cohort 2 included nine Indigenous women with RA aged 28-69 years. None of the participants were in the process of changing their RA therapy during the study period, their disease duration ranged from 4 months to 30 years, and they had been exposed to either diseasemodifying antirheumatic drugs (DMARDs) only $(n=4)$, DMARDs and biologics $(n=4)$, or no RA medication $(n=$ 1). Participants self-identified as First Nations, Plains Cree, or Indigenous.

\subsection{Cohort 1 Input Informing Initial Modifications}

Cohort 1 suggested the following modifications to the existing Early RA PtDA: (1) clarify medication names and routes of administration, (2) include Indigenous traditional healing practice options, (3) provide information on formulary coverage for Indigenous patients, (4) simplify text, and (5) include Indigenous images and use colors aligned with Canadian Indigenous community representation. The participants also suggested (6) incorporating evidence from Indigenous population or patient studies about medications and their effects and (7) providing translation of key words into Indigenous languages [17]. Based on these recommendations, TLF and VU modified the original decision aid, although the suggestions to include Indigenous-specific data in the decision aid could not be applied, due to lack of availability of this evidence, and it was decided that translation into Indigenous languages would occur prior to dissemination to specific communities. Table 1 summarizes the initial edits made.

\subsection{Cohort 2 Verifications and Additional Modifications}

The second cohort of participants affirmed alignment with the changes made to the original decision aid and suggested further adaptations to be made, outlined in Table 2, which we substantiate with the participants' quotations in the following subsections.

\subsubsection{Layout, Legibility, and Clarity}

Participants acknowledged that the formatting of the decision aid and the flow of information was "clear and pretty thorough" (cohort 2, participant [P] 9). The pictures and different sections of the decision aid advanced its clarity and enabled understanding. As stated by a participant, "Like, even picture-wise, it helps one to understand it and whatnot. And with-you know, like, different pinpoints that are right here ... it really helps. Like, it really breaks it down" (cohort 2, P4). Another mentioned, "The sections explain what the title is about, and I found it very organized" (cohort 2, P6). Participants noted that the language and wording used in the decision aid were simplified and concise, making the tool easy to read: "It's user-friendly ... it kind of breaks it down into layman's terms, because it's, like - you're not giving too much, like, higher medical terminology that's really hard to understand for the regular person, so it's - yeah, I think - and it's pretty neutral. I think it's okay" (cohort 2, P2).

Nonetheless, as much as they found the decision aid to be organized and clear, most participants indicated that the text used in the tool was not very legible. A participant stated, "[E]verything's good. It's just the words that are tiny" (P4) 


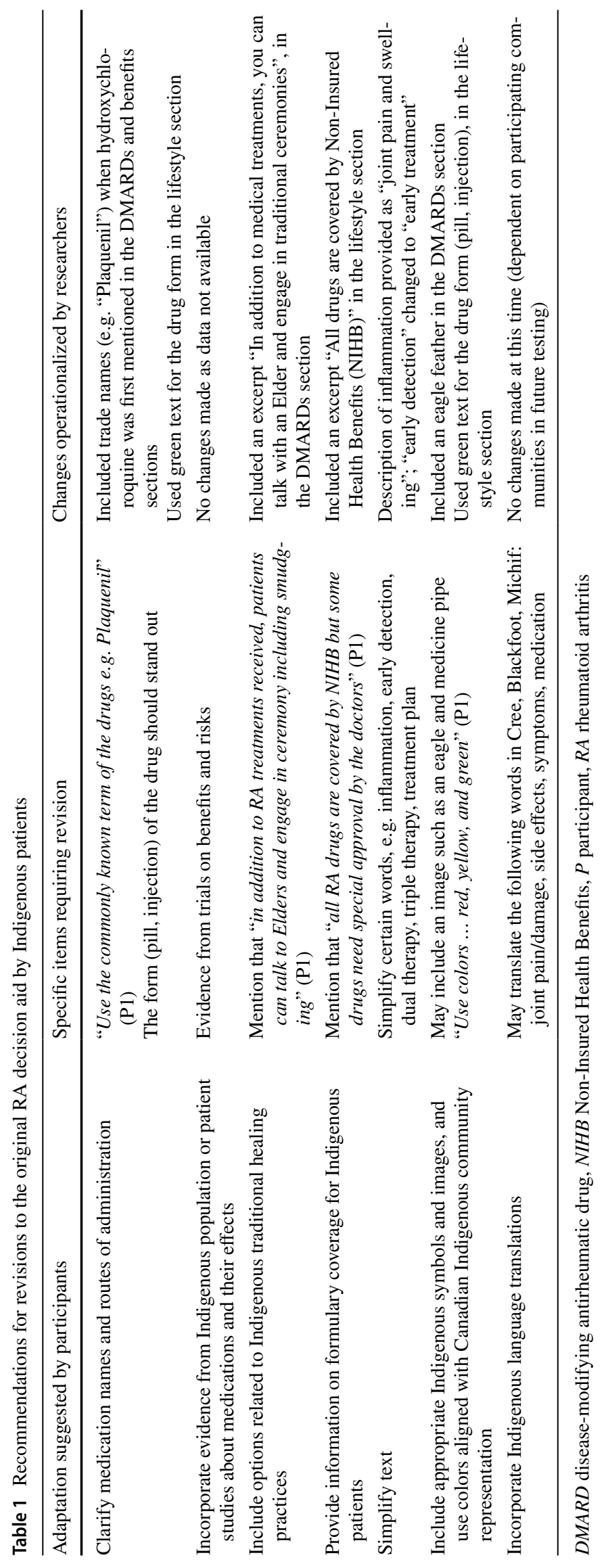




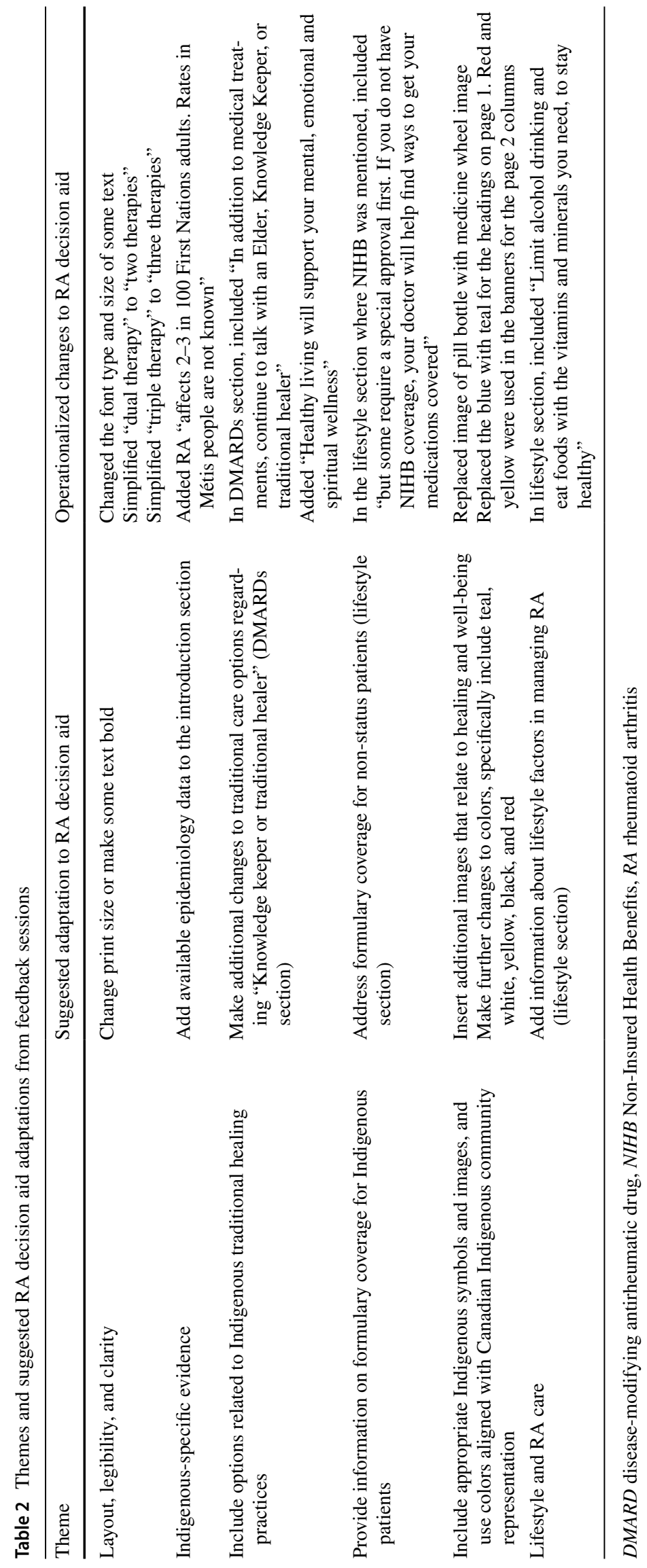


and another noted, "It's pretty clear if I really, you know, focus on it" (cohort 2, P9). Thus, a participant suggested, "Maybe a little bigger [text]" (cohort 2, P3), and another suggested bolding or unbolding some text to increase legibility of text. For example, a participant stated, "I'm just wondering, like, if the side effects have to be bolded, or should we bold, like, the medication itself?" (cohort 2, P2).

\subsubsection{Traditional Care and Healing Practices}

Participants appreciated including Indigenous traditional healing practices as adjuncts to treatment in the decision aid, as one participant stated, "Well, I like how you put in you can talk to an elder and engage in traditional ceremonies. That's nice ... I really like that. You never see anything that puts traditional therapy or talk to an elder" (cohort 2, P3). Additional changes were suggested, particularly including holistic care components tailored to the population. A participant indicated, "[I]n Blackfoot, because that's where I'm from, there's a difference. So if I'm going to go get doctored, I would go to a healer ... An elder would be somebody who would, you know, not necessarily have those rights ... it would have made more, like, an impact if it, you know, talk with a traditional healer or knowledge keeper" (cohort 2, P8).

\subsubsection{Formulary Coverage for Non-status First Nations Patients}

Participants affirmed the value of including the information regarding Non-Insured Health Benefits (NIHB) in the decision aid. As stated, "I really like how you put 'All drugs are covered by Non-Insured Health Benefits.' That's really good ... that's good that that's in there" (cohort 2, P2). However, a participant raised a concern on providing clarity on coverage of medications for non-status Indigenous patients, noting, 'So when it says "Non-Insured Health Benefits," what does that mean?... Okay, what if you're still First Nations and don't have that?... Because I'm First Nations and don't have status' (cohort 2, P3). Based on this feedback, additional information regarding formulary coverage for non-status First Nations patients was added.

\subsubsection{Indigenous Imagery and Colors}

Participants generally appreciated the eagle feather image that was included in the DMARDs section of the modified decision aid. Nonetheless, they mentioned the need to include more images that Indigenous patients using the tool will relate to. A participant stated, "So maybe put, like..., some medicine wheels on there, or, like - you've got a feather on there already. Anything that relates to, like, healing and positivity and - you know, something happy that
First Nations could relate to so that it's, like - a healing type of an image or thing" (cohort 2, P2). Another suggested, " $A$ watermark that's Indigenous ... A watermark would be nice ... I would put either the medicine wheel ... or a feather ... a smudge bowl ... with sweetgrass" (cohort 2, P8). Participants acknowledged the value of using colors aligned with Canadian Indigenous community representation in the decision aid. Although there was a general impression that the decision aid was colorful, two participants expressed the need to include more Indigenous-specific colors. A participant stated, "The color is nice, but I think if you try and use our four colors, because when you look at - our colors are universal, right? So, when I see a document, I don't even look at the words, but I see the colors, I automatically focus on it ... Yeah. And so that'll say to me, my mind, okay, this has something to do with my people ... White, yellow, black, and red" (cohort 2, P8).

\subsubsection{Lifestyle and RA Care}

Participants commented on information related to lifestyle factors that influence arthritis care and management. Two participants were interested in viewing more information on diet and the use of alcohol in arthritis. One participant stated, 'But I would like, to know, how - the use of drugs and alcohol ... What's that word? Predisposes you even more to it, right? ... So, some kind of side wording, like, "The use of drugs and alcohol will..." (cohort 2, P8). The same participant went on to state that, "[B]ecause now that I'm older and I'm now paying attention to my health and to my diet, that's the biggest thing for me, is to connect my diet with the inflammation" (cohort 2, P8). Another participant suggested, "[A]dd more to ... the lifestyle" (cohort 2, $\mathrm{P} 4$ ).

\subsubsection{Potential Use of the Indigenous Adaptation of the Early RA PtDA}

Participants viewed the decision aid as a talking guide that would drive discussions with their doctor and advance communication and relationship building with health practitioners. Three participants indicated, "[B]ecause it's information" (cohort 2, P7), "II]f I were to read it, there's still bound to be question so..." (cohort 2, P4), and "I'd rather, you know, a [physician] tell me....walk through it with me" (cohort 2, P9). The decision aid was generally viewed as an informative tool that could be displayed at the doctor's office and referred to before, during, and after clinic appointments. As stated, "It looks like it belongs in a doctor's office. Like, in the waiting room, or when you're in the - if you're in their actual office where they have other stuff hanging up. They always have some health stuff hanging up ... or in a magazine or something" (cohort 2, P3). In addition, participants conveyed being empowered and validated by 
hearing and including the voices of First Nations' people in developing health decision tools. For example, in the words of a participant, "It's about time we get a say in what's going on with our First Nations" (cohort 2, P5). Another expressed "Relief, ... because I'm informed, because this reinforces my knowledge thus far, I have more confidence" (cohort 2, P8).

\subsection{Finalization of the Adaptation of a PtDA for RA Treatment for Use with Indigenous Patients}

Following data review, the research team made final revisions to address the additional recommendations and other changes that were not operationalized in the initial modification. This was presented to the First Nations person with lived experience of RA engaged in the study for final review and approval. The final version of the Indigenous Adaptation of the Early RA PtDA is presented in Fig. 1.

\section{Discussion}

We used an iterative process to acquire feedback on a PtDA for early RA treatment decisions for use with Indigenous patients. While the existing Early RA PtDA included valued medical content, clarifications and additions to increase relevancy to Indigenous community members were required, while incorporating visual and cultural elements to increase acceptability. The user-friendliness characteristics were welcome feedback, as this element is central in developing PtDAs [9] and is essential in supporting patients to acquire, understand, and use fact-based information [14].

Other researchers have determined that Indigenous patients are more likely to engage in SDM and have less anxiety when relevant health information is provided [20]. The participants' feedback has directly informed content adaptations to the Early RA PtDA. The cost of RA treatments and access to medication may be important for patient considerations and decision-making [21]; specific to the adapted Early RA PtDA, participants identified the importance of providing sufficient information on options of medication coverage available for Indigenous patients irrespective of their membership in status First Nations, non-status First Nations, or Métis communities. Also, additional information on lifestyle information for diet and alcohol use when living with RA was requested. In a population with high rates of overdoses and related mental health conditions, often associated with intergenerational trauma and experiences linked to the inequities in social determinants of health including poverty and food security [22], adding this content in the decision aid is of high relevance to Indigenous patients. Feedback obtained solidified the importance of the Indigenous adaptation of the Early RA PtDA connecting to culture, family, and traditions. Encouragement for engaging with elders, healers, and knowledge keepers as sources of wisdom and support was appreciated by the participants, as cultural practices and traditional medicines and teachings support identity and healing [23]. Healthcare providers demonstrating openness to incorporating traditional healing practices in care plans is an important action towards reconciliation, while also serving to eliminate the evidence hierarchy that is pervasive in Western medical systems.

Previous research has stated that specific PtDA design features are health literacy enabling, such as reducing the amount of text, simplifying medical jargon, and presenting essential information and numerical information in logical ways [24], while approaches to reduce language barriers should also be addressed [25]. The feedback on translation of key words into the specific Indigenous language of the end-user reinforces this advice; however, we add here that visual communication approaches are vital [26, 27]. Indigenous peoples are visual learners and relate with traditional images that promote health and well-being, as well as the use of specific colors when disseminating health information. Indigenous art and images can be a communication tool to assist in bridging the divide between non-Indigenous health systems and Indigenous worldviews and understanding of health decisions and information [28].

Building on the needs assessment findings [17] and reinforced in the guidance for an implementation strategy for PtDAs in rheumatology [5], additional participants in this study report that the PtDA would be best applied in dialogue with their healthcare practitioner. While PtDAs may improve knowledge and reduce decisional conflict among RA patients [3], their use alone is limited without a clinician who is supportive of SDM. Healthcare practitioners working with Indigenous patients should offer decision-coaching to enhance the engagement of Indigenous patients in the decision-making process [14]. Related research on developing a SDM strategy for use by Inuit people for cancer care showed the value of using a PtDA with peer support workers to prepare patients to engage in decision-making with cancer care providers [15]. Dialogue is an important and integral element in wellbeing and aligns with Indigenous teachings, where information is shared via conversation and stories. Conversation advances informed healthcare decision-making and empowers individuals who experience discrimination and marginalization within care systems [14].

\subsection{Strengths and Limitations}

The feedback received from Indigenous patients is highly applicable to adaptation of other PtDAs in rheumatology and other disciplines. A strength was the approach to iteratively adapt the existing PtDA, already informed by national perspectives, with Indigenous-patient feedback, in alignment with patient-oriented research approaches. Although we 


\section{Should you use Methotrexate only, Two Therapies or} Three Therapies in Combination for Rheumatoid Arthritis?

WHAT IS RHEUMATOID ARTHRITIS?

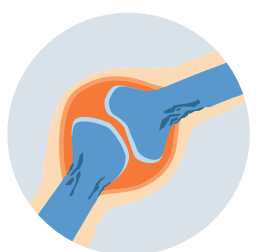

Rheumatoid Arthritis affects 1 in 100 adults. It affects $2-3$ in 100 First

Nations adults. Rates in Métis people are not known. It causes joint pain and swelling. Inflammation can also affect other organs including the lungs, nerves, eyes, or heart.

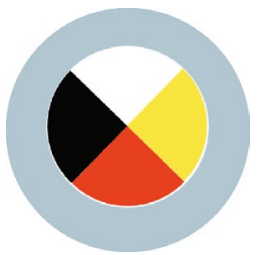

While there is no cure, there are very effective treatments for Rheumatoid Arthritis. Healthy living will support your mental, emotional and spiritual wellness.

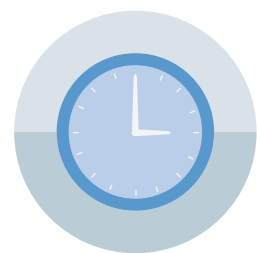

Early treatment is very important to stop joint damage.

\section{Disease Modifying Anti-Rheumatic Drugs (DMARDs)}

DMARDs help stop joint pain and swelling and prevent joint damage.

Treatments are adjusted to find the ones that work best for you.

DMARDs typically take 6-12 weeks to work. Other treatments can be used to control your symptoms while they have a chance to work.

All drugs are covered by Non-Insured Health Benefits (NIHB), but some require a special approval first. If you do not have NIHB coverage, your doctor will help find ways to get your medications covered.

If you are breastfeeding, pregnant or planning a pregnancy, please discuss this with your doctor. Methotrexate cannot be used in pregnancy.

THIS DECISION AID IS FOR YOU IF:

- You are age 16 or older

- Have a new diagnosis of active rheumatoid arthritis $\theta / \infty$ Methotrexate only

Methotrexate works well on its own and is taken once a week (pill or injection).

Q/

+ Methotrexate may work better when taken with another drug called Hydroxychloroquine (Plaquenil).

এ/

+ Methotrexate works best when given with two other

+() drugs: Hydroxychloroquine and Sulfasalazine.

None of the above

Talk to your doctor about other options.

In addition to medical treatments, continue to talk with an Elder, Knowledge Keeper, or traditional healer and engage in traditional ceremonies.

WHY IS THIS DISCUSSION IMPORTANT?

All of these options work well and are safe. Each choice has different chances of helping your joint pain and swelling, different side effects, and number of daily pills. It is important that your doctor knows what matters most to you to help you decide the best option.

Fig. 1 Adapted early rheumatoid arthritis patient decision aid

may have alternatively proceeded with creating an entirely new PtDA, this may have placed additional research burden on the participants. A potential limitation is the representativeness of the participants, who were all women and were uniquely urban residents. However, our participants did represent various Indigenous communities, as well as a range of ages, disease duration, and exposures to RA medication, and thus were still a diverse group. Investments to translate key words to particular Indigenous languages are a future action item once locales for testing and implementation of 


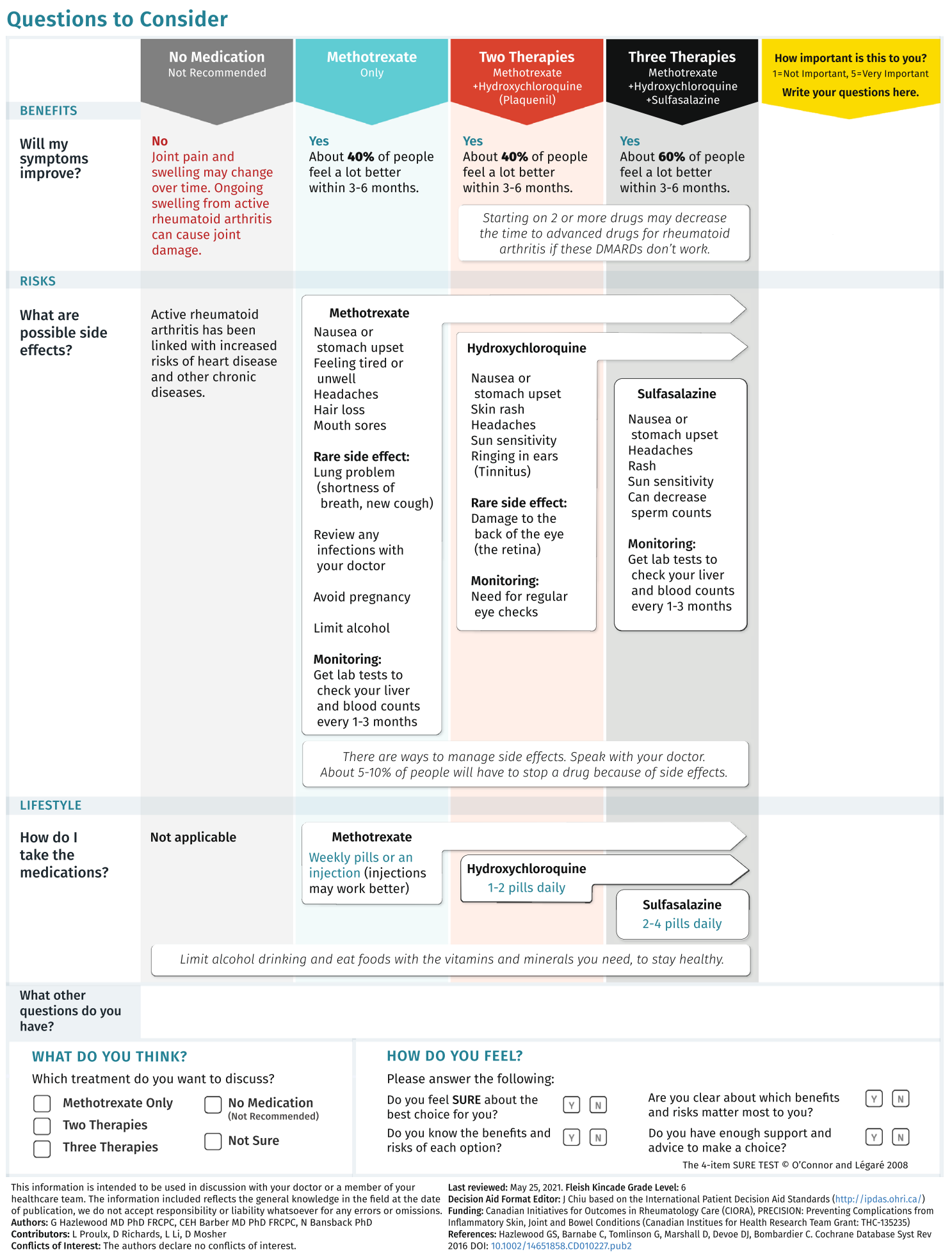

Fig. 1 (continued)

the Early RA PtDA are identified. Indigenous-specific data on efficacy and adverse effects of treatment would be beneficial for populating the decision aid estimates but are not currently available. Further research that involves testing the
Early RA PtDA for feasibility and acceptability, specifically in contrast to the original Early RA PtDA, would provide direct evidence on its effectiveness in promoting SDM in RA care for Indigenous patients. 


\section{Conclusion}

In an iterative process to adapt the Early RA PtDA, a modified version was produced to meet the decision-making needs of Indigenous patients with RA. Incorporating Indigenous-specific adaptations in the design of PtDAs may increase use and relevancy to support engagement in treatment decisions, thereby supporting health-equity-oriented health service interventions.

Supplementary Information The online version contains supplementary material available at https://doi.org/10.1007/s40271-021-00546-8.

Acknowledgements We appreciate the collaboration of Siksika Health Services in earlier phases of the research and the Elbow River Healing Lodge in Calgary who permitted patient recruitment from their centers.

\section{Declarations}

Funding Umaefulam: Eyes High Postdoctoral Scholarship, University of Calgary. Hazlewood: CIHR New Investigator. Barber: Arthritis Society's Stars Career Development Award funded by the Canadian Institutes of Health Research-Institute of Musculoskeletal Health and Arthritis STAR-19-0611/CIHR SI2-169745. Barnabe: Canada Research Chair, Rheumatoid Arthritis and Autoimmune Diseases, CIHR and a CIHR Foundation Scheme Grant.

Conflict of interest The authors declare that they have no conflict of interest.

Data availability Not applicable.

Code availability Not applicable.

Author contributions $\mathrm{VU}$ and $\mathrm{CB}$ contributed to the adaptation study conception and design. GH, NB, and CEHB developed the initial patient decision aid. All authors contributed to material preparation and data collection and analysis. All authors contributed to the writing of the manuscript and read and approved the manuscript and consent to publication.

Ethics approval Approval was obtained from the Conjoint Health Research Ethics Board of the University of Calgary, Canada (ethics approval number: REB19-1176).

Consent to participate Participation in this study was voluntary and written consent was obtained from all study participants.

Open Access This article is licensed under a Creative Commons Attribution-NonCommercial 4.0 International License, which permits any non-commercial use, sharing, adaptation, distribution and reproduction in any medium or format, as long as you give appropriate credit to the original author(s) and the source, provide a link to the Creative Commons licence, and indicate if changes were made. The images or other third party material in this article are included in the article's Creative Commons licence, unless indicated otherwise in a credit line to the material. If material is not included in the article's Creative Commons licence and your intended use is not permitted by statutory regulation or exceeds the permitted use, you will need to obtain permission directly from the copyright holder. To view a copy of this licence, visit http://creativecommons.org/licenses/by-nc/4.0/.

\section{References}

1. Légaré F, Thompson-Leduc P. Twelve myths about shared decision making. Patient Educ Couns. 2014;96(3):281-6.

2. Feenstra B, Lawson ML, Harrison D, Boland L, Stacey D. Decision coaching using the Ottawa family decision guide with parents and their children: a field testing study. BMC Med Inform Decis Mak. 2015;15(1):1-10.

3. Barton JL, Trupin L, Schillinger D, Evans-Young G, Imboden J, Montori VM, et al. Use of low-literacy decision aid to enhance knowledge and reduce decisional conflict among a diverse population of adults with rheumatoid arthritis: results of a pilot study. Arthritis Care Res. 2016;68(7):889-98.

4. Cochrane Musculoskeletal. Decision Aids. 2019. https://muscu loskeletal.cochrane.org/decision-aids. Accessed Jul 232019.

5. Barber CEH, Spencer N, Bansback N, Zimmermann GL, Li LC, Richards DP, et al. Development of an implementation strategy for patient decision aids in rheumatoid arthritis through application of the behavior change wheel. ACR Open Rheumatol. 2021;3(5):312-23.

6. Bansback N, Li LC, Lynd L, Bryan S. Development and preliminary user testing of the DCIDA (Dynamic computer interactive decision application) for "nudging" patients towards high quality decisions. BMC Med Inform Decis Mak. 2014;14(1):1-13.

7. Hazlewood GS, Marshall DA, Barber CEH, Li LC, Barnabe C, Bykerk $\mathrm{V}$, et al. Using a discrete-choice experiment in a decision aid to nudge patients towards value-concordant treatment choices in rheumatoid arthritis: a proof-of-concept study. Patient Prefer Adherence. 2020;14:829-38.

8. Hazlewood GS, Barnabe C, Tomlinson G, Marshall D, Devoe D, Bombardier C. Methotrexate monotherapy and methotrexate combination therapy with traditional and biologic disease modifying antirheumatic drugs for rheumatoid arthritis: abridged Cochrane systematic review and network meta-analysis. BMJ. 2016;353:i1777.

9. Elwyn G, O'Connor A, Stacey D, Volk R, Edwards A, Coulter A. Developing a quality criteria framework for patient decision aids: online international Delphi consensus process. Br Med J. 2006;333(7565):417-9.

10. Dugas M, Trottier MÈ, Chipenda Dansokho S, Vaisson G, Provencher $\mathrm{T}$, Colquhoun $\mathrm{H}$, et al. Involving members of vulnerable populations in the development of patient decision aids: a mixed methods sequential explanatory study. BMC Med Inform Decis Mak. 2017;17(1):1-11. https://doi.org/10.1186/ s12911-016-0399-8.

11. Barnabe C, Jones CA, Bernatsky S, Peschken CA, Voaklander $\mathrm{D}$, Homik J, et al. Inflammatory arthritis prevalence and health services use in the first nations and non-first nations populations of Alberta, Canada. Arthritis Care Res. 2017;69(4):467-74.

12. Nagaraj S, Barnabe C, Schieir O, Pope J, Bartlett SJ, Boire G, et al. Early Rheumatoid arthritis presentation, treatment, and outcomes in aboriginal patients in Canada: a Canadian early arthritis cohort study analysis. Arthritis Care Res. 2018;70(8):1245-50.

13. Enard KR, Mullen PD, Kamath GR, Dixon NM, Volk RJ. Are cancer-related decision aids appropriate for socially disadvantaged patients? A systematic review of US randomized controlled trials. BMC Med Inform Decis Mak. 2016;16(64):1-15. https://doi.org/ 10.1186/s12911-016-0303-6.

14. Jull J, Giles A, Minwaashin L, Boyer Y, Stacey D. Cultural adaptation of a shared decision making tool with Aboriginal women: a qualitative study. BMC Med Inform Decis Mak. 2015;15(1):1-13.

15. Jull J, Hizaka A, Sheppard AJ, Kewayosh A, Doering P, Macleod $\mathrm{L}$, et al. An integrated knowledge translation approach to develop a shared decision-making strategy for use by Inuit in cancer care : a qualitative study. Curr Oncol. 2019;26(3):1-27. 
16. Loyola-Sanchez A, Hazlewood G, Crowshoe L, Linkert T, Hull PM, Marshall D, et al. Qualitative study of treatment preferences for rheumatoid arthritis and pharmacotherapy acceptance: indigenous patient perspectives. Arthritis Care Res (Hoboken). 2020;72(4):544-52.

17. Umaefulam V, Fox T, Barnabe C. Decision needs and preferred strategies for shared decision making in rheumatoid arthritis: perspectives of Canadian urban indigenous women. Arthritis Care Res (Hoboken). 2021;20:10-3.

18. O'Connor A, Jacobsen M. Workbook on developing and evaluating patient decision aids. Ottawa Health Research Institute. 2003. http://decisionaid.ohri.ca/docs/develop\%5Cn/Develop_DA.pdf.

19. Nowell LS, Norris JM, White DE, Moules NJ. Thematic analysis: striving to meet the trustworthiness criteria. Int J Qual Methods. 2017; 16(1):1-13.

20. Groot G, Waldron T, Barreno L, Cochran D, Carr T. Trust and world view in shared decision making with indigenous patients: a realist synthesis. J Eval Clin Pract. 2020;26(2):503-14.

21. Binder-Finnema P, Dzurilla K, Hsiao B, Fraenkel L. Qualitative exploration of triangulated, shared decision-making in rheumatoid arthritis. Arthritis Care Res (Hoboken). 2019;71(12):1576-82.

22. National Collaborating Centre for Aboriginal Health. State of knowledge of aboriginal health: a review of Aboriginal Public Health in Canada. Prince George: National Collaborating Centre for Aboriginal Health; 2012.

23. Viscogliosi C, Asselin H, Basile S, Borwick K, Couturier Y, Drolet MJ, et al. Importance of Indigenous elders' contributions to individual and community wellness: results from a scoping review on social participation and intergenerational solidarity. Can J Public Heal. 2020;111(5):667-81.

24. Muscat DM, Shepherd HL, Nutbeam D, Trevena L, McCaffery KJ. Health literacy and shared decision-making: exploring the relationship to enable meaningful patient engagement in healthcare. J Gen Intern Med. 2021;36(2):521-4.

25. Barton JL, Koenig CJ, Evans-Young G, Trupin L, Anderson J, Ragouzeos D, et al. The design of a low literacy decision aid about rheumatoid arthritis medications developed in three languages for use during the clinical encounter. BMC Med Inform Decis Mak. 2014;14(104):1-14.

26. Bilodeau G, Witteman H, Légaré F, Lafontaine-Bruneau J, Voyer $\mathrm{P}$, Kröger E, et al. Reducing complexity of patient decision AIDS for community-based older adults with dementia and their caregivers: multiple case study of decision boxes. BMJ Open. 2019;9(5):1-19.

27. Durand MA, Alam S, Grande SW, Elwyn G. "Much clearer with pictures": using community-based participatory research to design and test a Picture Option Grid for underserved patients with breast cancer. BMJ Open. 2016;6:e010008. https://doi.org/10.1136/ bmjopen-2015-010008.

28. Muirhead A, Leeuw S De. Art and wellness: the importance of art for Aboriginal peoples' health and healing. 2012. https://artsh ealthnetwork.ca/sites/default/files/art_wellness_en_web.pdf. 